\title{
Constructing One-Dimensional Continuous Models from Two-Dimensional Discrete Models of Medical Implants
}

\author{
Alicia Prieto-Langarica*, Hristo V. Kojouharov ${ }^{\dagger}$, Liping Tang $\ddagger$ \\ * Department of Math. and Statistics, Youngstown State Univ., Youngstown, OH, USA \\ Email: aprietolangarica@ysu.edu \\ $\dagger$ Department of Mathematics, The Univ. of Texas at Arlington, Arlington, TX, USA \\ Email: hristo@uta.edu \\ $\ddagger$ Department of Bioengineering, The Univ. of Texas at Arlington, Arlington, TX, USA \\ Email: ltang@uta.edu
}

Received: 17 July 2012, accepted: 04 September 2012, published: 08 October 2012

\begin{abstract}
Medically implanted devices are becoming increasingly important in medical practice. Over 4 million people in the United States have long-term biomedical implants. However, many medical implants have to be removed because of infection or because their protein coating causes excessive inflammation and decrease in the immune system response. In this work, a discrete twodimensional model of blood cells and bacteria interactions on the surface of a medical implant is transformed into a discrete one-dimensional model. This one dimensional model is then upscaled into a partial differential equation model. The results from the discrete two-dimensional model and the continuous one-dimensional model are then compared for different protein coating mixtures. Two medical treatment alternatives are also explored and the two models are compared again.
\end{abstract}

Keywords-upscaling; medical implants; modeling; cellular automata

\section{INTRODUCTION}

\section{A. Biological Background}

Medically implanted devices are becoming increasingly important in medical practice [18]. Since the first applications of biomaterials in medicine, infections represent the most important complications, which still limit the unrestricted use of biomaterials in humans [3]. Implant-associated infections account for nearly $50 \%$ of the estimated 2 million nosocomial infections in the United States each year, [3]. The most common medical implant infections are due to Staphyloccocus epidermidis [17], which is a bacterial colonizer of the skin and mucous membranes of humans and other mammals [10]. S. epidermidis can lead to a wide variety of complications including inflammation, thrombosis, infections and fibrosis [18]. These complications have a direct effect on the stability of the implanted device because they trigger immune responses, including a rapid accumulation of phagocytic cells [18].

If the immune system is not able to eradicate $S$. epidermidis during the first several hours after it has entered the body then biofilm formation is likely to commence. Biofilms represent the most prevalent type of microbial growth in nature and are crucial to the development of clinical infections. They can serve as a nidus for disease and are often associated with high-level antimicrobial resistance of the associated organisms [7]. Studies suggest that biofilms are present on the surface of the implant as early as 16 hours after implantation [4]. However young biofilms are more vulnerable to phagocytic cells than mature ones which have been growing for more than 48 hours [4]. In addition, most antibiotics are only effective against the fast growing bacteria which reside in the outer layers of the biofilm, 


\section{A. Prieto-Langarica et al., Constructing One-Dimensional Continuous Models...}

while the slow growing bacteria deep inside of the biofilm formation tend to be spared and to persist in the body [2].

Therefore, it is critical that the immune system destroys the majority of the bacteria before a biofilm begins to form. Of all the types of phagocytic cells, the most important to the immune system's defense against $S$. epidermidis are the white blood cells called neutrophils. In order to attack the S. epidermidis growing on medical implants, neutrophil cells adhere to the surface of the device and move towards the bacterial formations [18]. Previous studies have indicated that the rate of neutrophil locomotion is influenced by adhesive forces between the cells and the substratum [6].

Fibrinogen and albumin are two of the most commonly used protein coatings on medically implanted devices. Fibrinogen facilitates a strong attachment between neutrophils and the implant since it is readily recognized as a malign substance by the immune system. However, fibrinogen also works as a distraction to the neutrophils because the phagocytes place themselves in one spot attacking the fibrinogen covered implant and move very slowly towards the bacteria [8, 16]. In contrast, albumin is not recognized by the phagocytes as a malign substance and hence the neutrophils cells can move freely around the implant.

Another important distinction between albumin and fibrinogen is the amount of neutrophils each protein coating attracts. Experimental studies suggest that two groups of chemokines macrophage inflammatory protein (MIP) and monocyte chemoattactant protein (MCP) appear to play a major role in phagocyte-implant interactions [18]. By releasing chemokines, the neutrophil cells present on the surface of the implant are able to attract more neutrophils to the site. These chemotactic interactions create waves of incoming phagocytic cells, which aid in the fight against the bacterial infection. While fibrinogen covered implants are interpreted as a threat to the body and many phagocytes are attracted to them, the albumin coated implant is not perceived as a threat and thus fewer phagocytes are present to fight the infection.

\section{B. Mathematical Model}

A discrete two-dimensional Cellular Automata (CA) model [9] that describes the interactions between neutrophil cells and S. epidermidis cells on the surface of an implant was previously developed [14]. A cellular automaton consists of a regular grid, where each site in the lattice can be in one of a finite number of possible states updated synchronously in discrete time steps according to local, identical rules [9]. A set of rules for the movement of the cells and the growth of the bacteria is given for the two different types of protein coatings. The amounts of albumin and fibrinogen in the mixture are allowed to be varied, since they have different effects on the speed of the neutrophils and their ability to control a bacterial infection.

In the CA model neutrophil cells move with greater probability towards larger bacterial concentrations. The model is divided into three parts. The first part simulates the complex S. epidermidis-neutrophils interactions between 4 and 20 hours after the implant is introduced into the body. At this early stage, reproduction of bacteria and early bacterial community formation triggers the immune system response. A series of chemotactic waves of neutrophil cells are then incorporated into the system and are considered in the model. The second part of the model simulates the system dynamics after the $S$. epidermidis have started forming a biofilm, which takes place between the 20 and the 52 hours. During this part of the simulation, bacteria experience an increase in the reproduction rate while the immune system response gradually decreases effectiveness as the biofilms become stronger. The last part of the model, after the initial 52 hours, the immune system can no longer fight $S$. epidermidis since they are all gathered in fully formed strong biofilms.

An important aspect of the CA model is the different scales being used. Two different grids are considered: a $12 \times 12$ grid for the neutrophil cells and a $144 \times 144$ grid for the $S$. epidermidis since neutrophil cells are 12 times larger in size than S. epidermidis cells. However, the two-dimensional CA only models $0.1 \%$ of the total experimental implant area. Larger areas become almost impossible to simulate since running the $\mathrm{CA}$ model is computationally very expensive. In order to model larger areas continuous model needs to be used. By adding the elements on each column and expressing the result on a line grid, the model is then transform into a one-dimensional discrete model. Using the upscaling method described in [11, 12, 13] the one-dimensional discrete model is then upscaled into a continuous partial differential equation (PDE) model by considering the transition probabilities of each site from one state to another and then taking limits as space and time steps tend to zero.

Using both models, the discrete two-dimensional model and the continuous one-dimensional model, a variety of mixtures of fibrinogen and albumin implant coatings are examined in order to maximize the effec- 


\section{A. Prieto-Langarica et al., Constructing One-Dimensional Continuous Models...}

tiveness of the immune system response [14]. Finding the optimum amounts of each of these two proteins can help the immune system destroy most of the bacteria before they start to form biofilm communities. This can reduce the number of rejections of medically implanted devices and drastically improve the ability of the body's immune system to combat bacterial infections. The presented simulations can also be used to help determine the appropriate amount of antibiotics to use over the implant area so that an $S$. epidermidis infection can be successfully controlled, as well as to predict what can happen if biofilm formation is prevented.

\section{From Two-Dimensions to OnE-Dimension}

Let $u^{0}(a, b)$ be the initial number of neutrophil cells, at position $(a, b)$ in the grid and $u^{n}(a, b)$ be the expected number of neutrophil cells at position $(a, b)$ after $n$ time steps. Also let $w^{0}(x, y)$ be the initial number of $S$. epidermidis cells, at position $(x, y)$ in the grid and $w^{n}(x, y)$ be the expected number of $S$. epidermidis cells at position $(x, y)$ after $n$ time steps.

As a first step in constructing a one-dimensional discrete model, the initial distributions of the neutrophil cells and the $S$. epidermidis cells are added in each column of the grid as follows:

$$
\begin{aligned}
\hat{u}^{n}(a) & =\sum_{i=1}^{12} u^{n}(a, i), \\
\hat{w}^{n}(x) & =\sum_{i=1}^{144} w^{n}(x, i) .
\end{aligned}
$$

This creates the corresponding one-dimensional initial density profiles (Figure 1). The rules for movement, the rates of bacterial reproduction, addition of neutrophils into the system, and neutrophils killing bacteria are all kept the same. A similar approach has been taken before in the literature [15], for transforming a two-dimensional discrete model into a one-dimensional discrete model.

Next, the corresponding one-dimensional continuous model is constructed. In order to do this, transition probabilities are defined for the state of each point in the grid creating a discrete description of all undergoing processes [11]. Taking limits as the time step and the mesh size tend to zero yields the following system of partial differential equations:

$$
\begin{aligned}
\frac{\partial u}{\partial t} & =D \frac{\partial^{2} u}{\partial x^{2}}-\frac{\partial}{\partial x}(V u)+(k w+a) u, \\
\frac{\partial w}{\partial t} & =D_{w} \frac{\partial^{2} w}{\partial x^{2}}+\left(r-k_{e} u\right) w,
\end{aligned}
$$

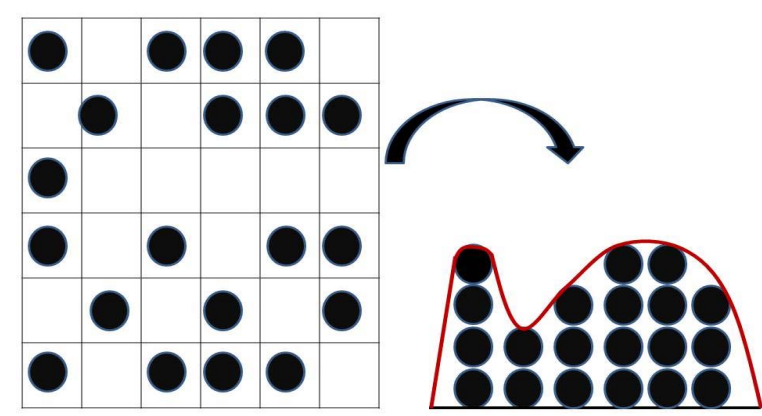

Fig. 1. 2-D to 1-D conversion through column addition.

where $V$ and $D$ are the advection and diffusion coefficients, respectively, in the neutrophils equation, $k$ is the rate at which neutrophil cells call on other neutrophil cells depending on the presence of $S$. epdidermidis while $a$ is the rate at which neutrophil cells call on other neutrophil cells independently of the presence of $S$. epidermidis. $D_{w}$ is the diffusion coefficient in the $S$. epidermidis equation, $r$ is the growth rate of $S$. epidermidis, and $k_{e}$ is the rate at which neutrophil cells kill $S$. epidermidis cells.

\section{NUMERICAL SimUlations}

The one-dimensional continuous model (2) is numerically solved and the results are compared with the results obtained after running 100,000 simulations of the CA model accounting for the amount of bacteria left in each simulation after $T=20, T=52$ and $T=76$ hours. A simulation is considered effective if at least $99 \%$ of the implant area is free of bacteria coverage after 76 hours. Figure 2 shows the percentage of effective simulations for a variety of mixtures of protein coatings of the implant with albumin percentages between $0 \%$ and $100 \%$ in $10 \%$ increments ( $\%$ of fibrinogen $=100-\%$ of albumin) after $T=20, T=52$ and $T=76$ hours for both the discrete 2-D and continuous 1-D models.

As Figure 2 shows, mixtures with either high albumin or low albumin percentages yield a high percentage of ineffective simulations. However, two treatment strategies can be used to improve result for all protein mixtures: (1) medical devices can be pre-coated with antibiotics before implantation; or (2) biofilm formation can be blocked [5]. Accordingly, the mathematical model is modified to include both strategies:

- The effect of antibiotics is included in the model by randomly selecting a fixed percentage of bacteria every certain amount of time and eliminating it 

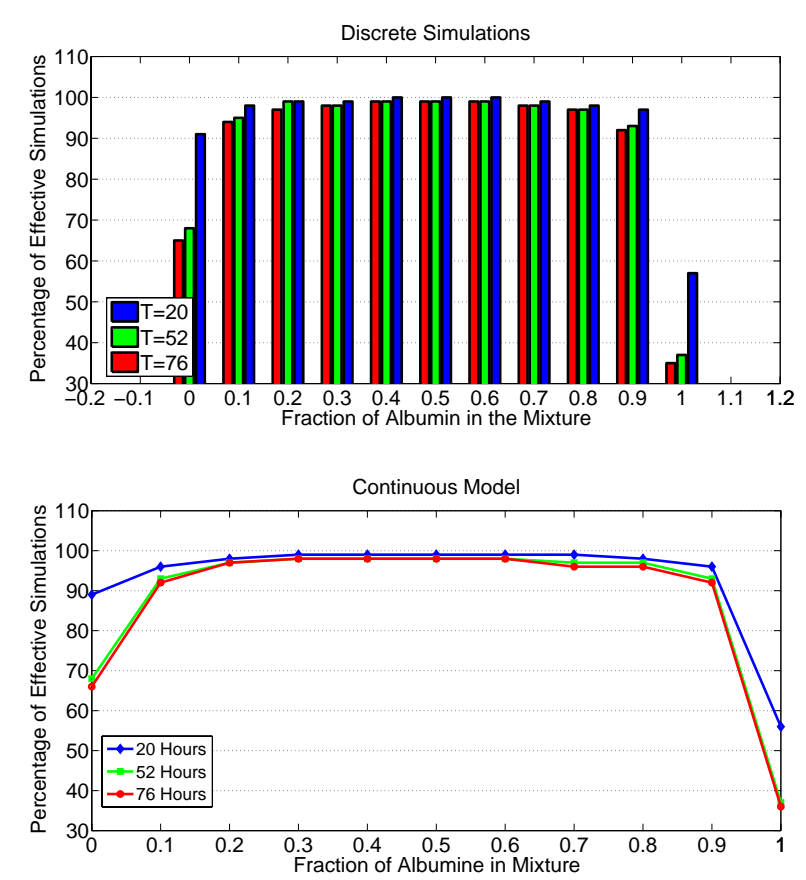

Fig. 2. Comparison of the discrete cellular automata 2-D model (above) and the continuous 1-D differential equation model (below) for $T=20, T=52$ and $T=76$.

from the implant. The percentage of bacteria to be eliminated is a parameter in the model and can be modified to represent different antibiotics. Figure 3 shows the effects of pre-coating the implant with a sample antibiotic for all different protein coating mixtures.

- The second strategy entails disrupting the agr system to prevent bacterial attachment and therefore avoid biofilm formation. No biofilm formation translates into treating both parts in the model, the 20-to-76 hour and the 4-to-20 hour, in a similar way, i.e., all bacteria are treated as free bacteria and neutrophils kill bacteria at the same constant rate throughout the entire simulation. The results of disrupting the agr system are shown in Figure 4

As it can be seen in Figures 3 and 4 , there is a very good agreement between the two-dimensional discrete $\mathrm{CA}$ model and the one-dimensional continuous PDE model when either of the two treatment strategies is used.

A comparison of the cpu time used in Matlab $R$ to compute the corresponding numerical solutions is presented in Table 1 T The PDE model was solved using a finite difference method [1] while the CA model was run 100,000 times. As shown on Table I. the two-dimensional discrete CA model simulations takes
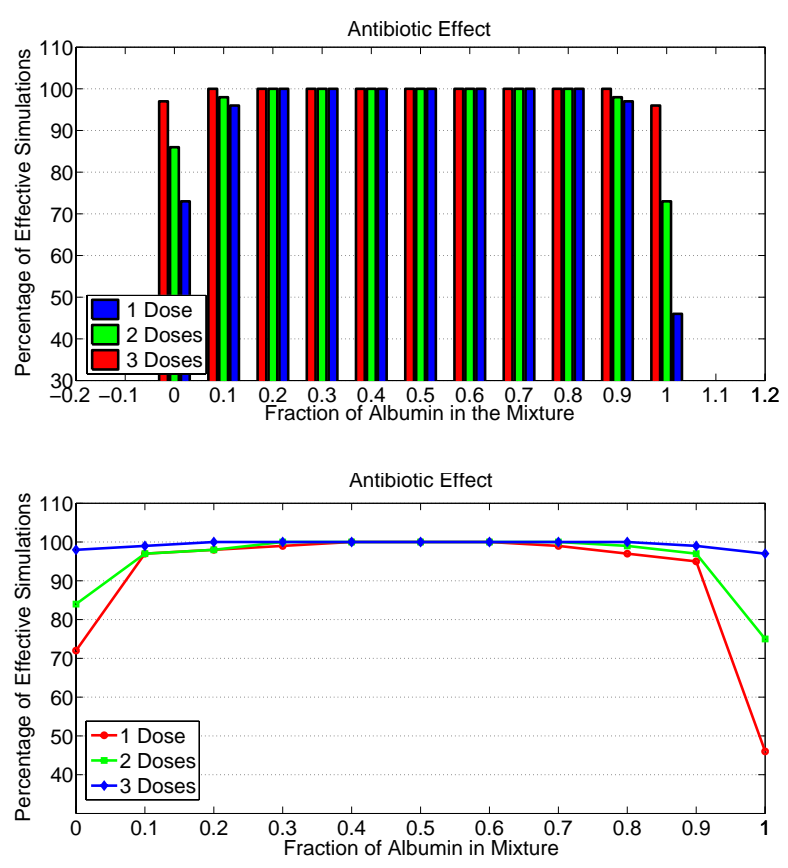

Fig. 3. Effects of different doses of antibiotics on bacterial growth on the surface of an implant. Discrete (above) and continuous (below) models results for $T=76$.

overwhelmingly longer time to run compared to the numerical solution of the one-dimensional continuous PDE model.

\section{CONCLUSION}

In this work, a two-dimensional discrete CA model and a one-dimensional PDE model for the interactions between neutrophils and S. epidermidis on the surface of a medical implant under different protein coating were explored. The PDE model was solved numerically and the results compared with 100,000 runs of the CA model. Both models where used to determine the protein coating mixture that will allow the immune system to eradicate $S$. epidermidis within 74 hours after implantation. The models were then modified to include the effect of pre-coating the implants with antibiotics and

\begin{tabular}{lccc}
\hline Problem & Figure & PDE Model & CA Model \\
\cline { 3 - 4 } & & $\mathrm{cpu}(\mathrm{h})$ & $\mathrm{cpu}(\mathrm{h})$ \\
\hline Implant Experiment & 2 & 0.0231 & 42.57 \\
Antibiotic Experiment & $\mathbf{3}$ & 0.0294 & 49.27 \\
No Biofilm Experiment & $\mathbf{4}$ & 0.0227 & 41.98 \\
\hline
\end{tabular}

TABLE I

COMPARATIVE COST OF THE CONTINUOUS AND DISCRETE MODELS IMPLEMENTED ON AN INTEL ${ }^{\circledR}$ CORE $^{\mathrm{TM}}$ CPU $2.53 \mathrm{GHz}$. 

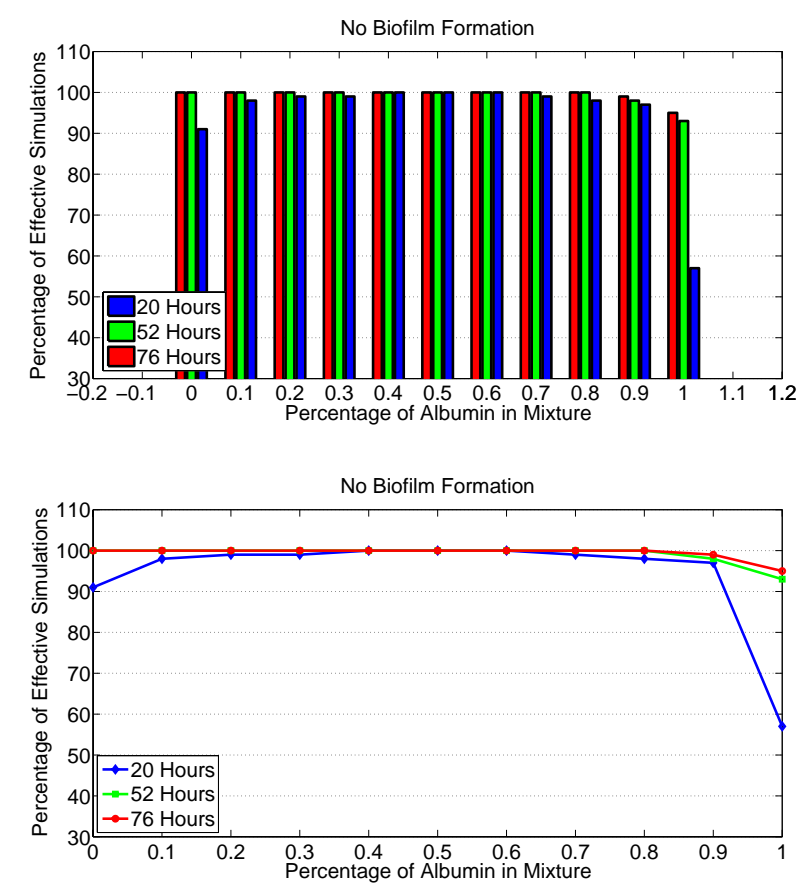

Fig. 4. Effect of no biofilm formation on bacterial growth on the surface of an implant. Discrete (above) and continuous (below) models results for $T=20, T=52, T=76$.

disturbing biofilm formation as two different treatment strategies.

The two-dimensional CA discrete model represents very accurately the medical implant system of interest. However, as can be seen in Table I] running 100,000 iterations of the discrete model is computationally expensive and it models only $0.1 \%$ of the implant area. Using the upscaling method described in [11] together with the conversion from two-dimensions to one-dimension, an efficient partial differential equations model can be constructed. The PDE model uses the correct parameters from the CA model making it both biologically accurate and very efficient to solve numerically as shown in Table II. Therefore, constructing a one-dimensional continuous model using what is presented in this paper can be as accurate in representing the biology of the problem and much more computationally efficient making it more feasible to use in practice.

\section{REFERENCES}

[1] W. F. Ames, Numerical Methods for Partial Differential Equations, 3rd ed., Academic Press, New York, 1992.

[2] J. Eberhard, Y. Efendiev, R. Ewing, A. Cunningham, "Coupled Cellular Models for Biofilm Growth and
Hydrodynamic Flow in a Pipe", Multiscale Comput. Engr., vol. 3 no. 4, pp. 499-516, 2005. http://dx.doi.org/10.1615/IntJMultCompEng.v3.i4. 70

[3] H. Gollwitzer, K Ibrahim, H. Meyer, W. Mittelmeier, R. Busch, A. Stemberger, "Antibacterial Poly(d,llactic acid) Coating of Medical Implants Using a Biodegradable Drug Delivery Technology", Antimicrob. Chemother., vol. 51 no. 3, pp. 585-591, 2003.

[4] F. Gunter, Wabnitz, P. Stroh, B. Prior, U. Obst, Y. Samstag, C. Wagner, M. Hansch,"Phagocytosis of Staphylococci Biofilms by Polymorphonuclear Neutrophils: S. Aureus and S. Epidermidis Differ with Regard to Their Susceptibility Towards the Host defense", Int. J. Artif. Organs., vol. 32 no. 9, pp. 565-573, 2009.

[5] K. Kong, C. Vuong, and M. Otto, "Staphylococcus quorum sensing in biofilm formation and infection", Int. J. Med. Microbiol., vol. 296 no.2-3, pp. 133139, 2006.

http://dx.doi.org/10.1016/j.ijmm.2006.01.042

[6] H. Keller, S. Barandun, P. Kristler, J. Ploem, "Locomotion and adhesion of neutrophil granulocytes: Effects of Albumin, Fibrinogen and Gamma Globulins Studied by Reflection Contrast Microscopy", Exp. Cell Res., vol. 122 no. 2, pp. 351-362 1979. http://dx.doi.org/10.1016/0014-4827(79)90311-2

[7] D. Kuhn, T. George, J. Chandra, P. Mukherjee, M. Ghannoum, "Antifungal Susceptibility of Candida Biofilms: Unique Efficacy of Amphotericin B Lipid Formulations and Echinocandins", Antimicrob. Agents. Chemother., vol. 46 no.6, pp. 17731780, 2002.

[8] R. Kuntz, W. Saltzman, "Neutrophil Motility in Extracellular Matrix Gels: Mesh Size and Adhesion Affect Speed of Migration”, Biophys. J., vol. 72 no. 3, pp. 1472-1480, 1997. http://dx.doi.org/10.1016/S0006-3495(97)78793-9

[9] D. Mallet, L. de Pillis, "A Cellular Automata Model of Tumor-Immune System Interactions", J. Theor. Biol., vol. 239 no. 3, pp. 334-350, 2006. http://dx.doi.org/10.1016/j.jtbi.2005.08.002

[10] M. Otto, "Staphylococcus Epidemidis — the 'Accidental' Pathogen", Nat. Rev. Microbiol., vol. 7 no. 8, pp. 555-567, 2009. http://dx.doi.org/10.1038/nrmicro2182

[11] A. Prieto-Langarica, H. Kojouharov, B. ChenCharpentier, "Upscaling from Discrete to Continuous Mathematical Models of two Interacting Populations", submitted, 2012. 
[12] A. Prieto-Langarica, H. Kojouharov, B. ChenCharpentier, "Discrete and Continuous Approaches to Modeling Cell Movement in the Presence of a Foreign Stimulus", Comput. Math. Appl., vol. 64 no. 3, pp. 167-174, 2012.

[13] A. Prieto-Langarica, H. Kojouharov, B. ChenCharpentier, "Mathematical Modeling of Chemoatractant Effects on Cell Movement", AIP Conf. Proc., vol. 1404 no. 1, pp. 184-192, 2011. http://dx.doi.org/10.1063/1.3659919

[14] A. Prieto-Langarica, H. Kojouharov, B. ChenCharpentier, L. Tang, "A Cellular Automata Model of Infection Control on Medical Implants", Appl. Appl. Math., vol. 6 no. 11, pp. 1741-1750, 2011.

[15] M. Simpson, K. Landman, B. Hughes, "MultiSpecies Simple Exclusion Processes", Physica A, vol. 388 no. 4, pp. 399-406, 2009. http://dx.doi.org/10.1016/j.physa.2008.10.038

[16] L. Tang, A. Lucas, J. Eaton, "Inflammatory Responses to Implanted Polymeric Biomaterials: Role of Surface-Adsorbed Immunoglobulin G', J. Lab. Clin. Med., vol. 122 no. 3, pp. 292-300, 1993.

[17] C. Vuong, M. Otto, "Staphylococcus Epidermidis Infections", Microbes. Infect., vol. 4 no. 4, pp. 481489, 2002. http://dx.doi.org/10.1016/S1286-4579(02)01563-0

[18] J. Xue, Gao, L. Tang, "Mathematical Modeling of Phagocyte Chemotaxis Toward and Adherence to Biomaterial Implants", BIBM 2007, pp. 302-307, 2007. 